\title{
PRÁCTICA REFLEXIVA COLECTIVA: UNA EXPERIENCIA DE DESARROLLO PROFESORAL EN EDUCACIÓN SUPERIOR
}

\section{COLLECTIVE REFLECTIVE PRACTICE: AN EXPERIENCE OF FACULTY DEVELOPMENT IN HIGHER EDUCATION}

Katina Isabel Camargo Ariza Ana Carolina Moreno Herrera

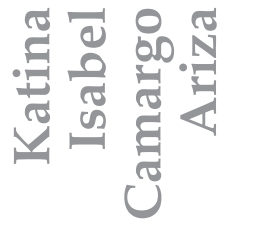

Magister en Educación. Asesora pedagógica en educación superior. Coordinadora Unidad de Formación Pedagógica Docente. Centro para la Excelencia Docente, Universidad del Norte (Barranquilla, Colombia).

katinac@uninorte.edu.co

Psicóloga- Asistente de investigación, Universidad del Norte (Barranquilla, Colombia) morenoca@uninorte.edu.co

\section{RESUMEN}

La práctica reflexiva como estrategia de formación docente plantea diferentes escenarios de trabajo. Uno de ellos se conoce como práctica reflexiva colectiva y permite a los participantes compartir su experiencia pedagógica y/o autoevaluar su práctica de aula, en un contexto de 
diálogo que establece condiciones para un ejercicio reflexivo entre pares. Este trabajo presenta los resultados del análisis de una experiencia de práctica reflexiva colectiva «Hablemos docencia», desarrollada por el Centro para la Excelencia de la Universidad del Norte (Barranqui1la, Colombia). En este espacio, los profesores participantes de diferentes áreas disciplinares, tienen la oportunidad de reflexionar sobre su práctica de aula en un escenario de diálogo grupal y colaborativo. La metodología empleada es de enfoque cuantitativo y ex post facto que caracteriza el efecto de la actividad «Hablemos docencia» en la práctica reflexiva de un grupo de profesores, teniendo en cuenta las dimensiones consideradas por Tsangaridou y O'Sullivan (1994).

Palabras clave: práctica reflexiva colectiva, desarrollo profesoral, docencia universitaria, educación superior.

\section{ABSTRACT}

Reflective practice as a professional development strategy allows us to envision different work scenarios. One of those is known as Collective Reflective Practice, which permits participants to share their pedagogical experience and/or self-assess their own practice in a context of dialogue that sets the conditions for a reflective peer practice. This paper shows the results of an analysis of a collective reflective practice called «Hablemos docencia» (Let's Talk about Teaching). An initiative developed by the Universidad del Norte's Center for Teaching Excellence in Barranquilla, Colombia. In this space, professors from different disciplines have the opportunity to reflect on their teaching practices in a group and collaborative setting. Grounded in insights by Tsangaridou and O'Sullivan (1994), we use a quantitative methodology and ex post facto approach, which characterizes the effect of «Hablemos docencia» on a group of professors.

Keywords: Collective Reflective Practice, Faculty Development, University Teaching, Higher Education. 


\section{INTRODUCCIÓN}

La práctica reflexiva colectiva se posiciona como una interesante variante para el pensamiento reflexivo docente. Esta propuesta promueve la autoevaluación de su práctica pedagógica y compartir experiencias que sirvan de base a otros docentes, como situaciones de referencia al enfrentarse a situaciones similares. Cuando se realiza sistemáticamente y con un propósito, los resultados pueden ser efectivos haciendo necesario caracterizar el impacto que genera este tipo de metodologías en los docentes.

Para esta tarea, es importante conocer los aportes teóricos que han dado origen a lo que se conoce hoy como "práctica colectiva reflexiva» y resaltar algunas investigaciones que se han llevado a cabo en torno a esta temática. Por esta razón, se realiza una revisión literaria de la práctica reflexiva con una línea de tiempo y relacionándolo con sus principales exponentes. De esta manera, es posible identificar cómo se ha implementado esta estrategia en las instituciones educativas y si los resultados son coherentes entre sí.

Este estudio es desarrollado por el Centro para la Excelencia Docente (CEDU), dependencia de apoyo al quehacer pedagógico de los docentes de la Universidad del Norte, mediante diferentes escenarios de formación y orientación pedagógica con el propósito de fomentar la excelencia e innovación en la enseñanza para facilitar el aprendizaje efectivo en el estudiante.

En cuanto a la metodología desarrollada, se implementó el modelo ex post facto que permite el estudio de hechos ocurridos en el pasado. Es una investigación de corte cuantitativo que tiene como objetivo evaluar el efecto que ha tenido el servicio «Hablemos docencia» en los docentes participantes durante un período académico determinado (20152017), a partir de los cambios metodológicos desde las dimensiones consideradas por Tsangaridou y O'Sullivan (1994).

Para la recolección y análisis de la información se empleó una entrevista semiestructurada que posteriormente fue transcrita para su respectivo análisis y categorización (focos y niveles). Este proceso dio 
como resultado que, efectivamente, los docente participantes en la actividad «Hablemos docencia» han desarrollado un nivel reflexivo básico que corresponde a describir lo que hacen los docentes considerando su experiencia previa; algunos lograron describir y justificar su quehacer docente teniendo en cuenta las investigaciones de aula realizadas, y finalmente, en algunas ocasiones, estos docentes pudieron describir, justificar y criticar su quehacer como profesores mediante la autoevaluación crítica.

\section{LA PRÁCTICA REFLEXIVA COMO UNA METODOLOGÍA TRANSFORMADORA}

En la actualidad, uno autor representativo sobre práctica reflexiva es John Dewey $(1967,1903,1997)$, un pedagogo estadounidense que plantea la idea de aprender mientras se realiza una acción; es decir, realizar cambios que emergen como producto de una concientización sobre la actividad que se realiza y no como un acto impulsivo. En otras palabras, establece una relación entre el pensamiento reflexivo y el proceso de aprendizaje. Dewey (1997, citado por Elías, Barcia, y Hernando, 2009) define el pensamiento reflexivo como «la consideración activa, consistente y cuidadosa de cualquier creencia o supuesta forma de conocimiento a la luz de los fundamentos que la sostienen y de las conclusiones a las que conduce» (p. 107).

Lo anterior, en el contexto de la práctica docente, considera que las metodologías de trabajo en el aula que emplea un profesor se fundamentan en un conocimiento previo, una teoría que respalde los contenidos y la manera en que deben ser enseñados o que respondan a las necesidades manifestadas por los estudiantes.

Dewey propone tres actitudes para establecer las condiciones que permitan el desarrollo del pensamiento reflexivo como práctica: 1) Apertura de mente (entendida como una actitud hacia el cambio y escucha activa ante los diferentes argumentos presentados, que permiten la toma de decisiones como profesional). 2) Responsabilidad intelectual (considerar las consecuencias de las propias decisiones y mantener una coherencia con los intereses 
personales). 3) Entusiasmo (motivación intrínseca que moviliza y facilita el proceso de aprendizaje) (Sánchez, Santos, y Ariza, 2005).

Siguiendo con la propuesta base de este autor, Donald Schön (1983, 1987) enfatiza el pensamiento reflexivo en la docencia, describe que la teoría es importante, pero al momento de llevar la teoría a la realidad no siempre se articulan de manera sinérgica. Lo que es posible es una reflexión en la acción y una reflexión sobre la acción; es decir, el primero concierne a una modificación de alguno de los elementos in situ y el segundo, corresponde a examinar - mediante el ensayo y el error - la práctica realizada, considerar sus consecuencias y replantear la acción (Elías, Barcia, y Hernando, 2009; Ortega, Nocetti, y Ortiz, 2015; Schön, (1987).

A pesar de la especificidad que propone Schön, autores como Elías, Barcia, y Hernando (2009), expresan que el ejercicio reflexivo - además de estar enmarcado en la acción manifestada o dirigida de afuera (el medio) hacia dentro (la interpretación) — debe considerar también las teorías implícitas o las concepciones de enseñanza que guían el acto de enseñar por parte del docente.

Las propuestas metodológicas de Dewey y Schön, planteadas en momentos diferentes, establecen que se requiere una situación detonante que involucre al docente, que lo lleve a la necesidad de reflexionar sobre ella. En palabras de Serrano (2005), «algunos de los seguidores de Dewey (como es el caso de Donald A. Schön, 1998) asumen que es la sorpresa, lo inesperado ante situaciones concretas, lo que desencadena en el individuo la posibilidad de reflexión» (p. 157).

Estas versiones propuestas, han sido actualizadas y contextualizadas de manera frecuente, puesto que las mismas investigaciones aportan nuevos conocimientos que se plantean como retos para la práctica docente. Es así como Domingo y Gómez (2014), conciben que la reflexión in situ debe manifestarse como un proceso analítico que permita al individuo aprender, teniendo en cuenta sus experiencias para promover una reconstrucción y / o resignificación personal de su conocimiento, mediante la revisión de las acciones realizadas. 
La reflexión, como elemento para la transformación social, es planteada por Habermas (1972, 1974), quien desarrolla una teoría que no solo describe las prácticas reflexivas, sino que señala los propósitos y resultados que deben tener para la construcción de una sociedad emancipada e igualitaria (Elías, Barcia, y Hernando, 2009, p. 109).

En este marco de referencia, propuesto por Habermas (1972, 1974) y Van Manen (1977), Tsangaridou y O'Sullivan (1994), proyectaron un estudio que describe cómo las estrategias pedagógicas reflectivas influencian la reflexión en futuros profesores. Esta investigación produjo una nueva clasificación para la práctica reflexiva: focos (técnico, contextual y reflexividad crítica) y niveles (descriptivo, justificativo y crítico) que se desarrollan de manera simultánea.

Con este sentido social, la reflexión puede prosperar en medio de la interacción entre pares o colegas, contribuyendo así a desplegar una visión más crítica y responsable para la construcción de nuevos aprendizajes en la dinámica de un diálogo reflexivo y crítico (Domingo, 2009).

Desde esta perspectiva de colaboración entre pares que sugiere Domingo (2009) — como una forma de generar un pensamiento reflexivo individual y compartido que genere cambios en las concepciones del docente hasta en su práctica de aula-, Catalán (2011), basado en la revisión de otros autores, propone una definición de «reflexión colectiva sistemática»:

[...] reflexión que realizan e intercambian los profesores, actuando como equipo de trabajo que ha recibido capacitación para analizar, intensificar y coordinar los procesos reflexivos en el abordaje de materias profesionales que les son relevantes (p. 207).

Esta propuesta es consistente con una disposición y una preparación por parte de los docentes participantes, que Dewey expone como actitudes. Así mismo, Cornejo (2011); De Vicente (1996) y Murillo (1999), citados por Catalán y Castro (2016), proponen que la participación de docentes en una misma reflexión puede incidir de manera positiva en su práctica pedagógica. 
Con base en lo anterior, y las nuevas necesidades de categorizar el concepto de práctica reflexiva, emerge una nueva propuesta que resulta de la integración de aportes de la filosofía, en cuanto a la definición de reflexión y la puesta en práctica de este de manera pertinente: la «competencia reflexiva» (CR).

Barnett (1992, citado por Domingo, 2009) señala que:

La CR es un medio para estimular a los estudiantes a que desarrollen la capacidad de observarse y de emprender un diálogo crítico con ellos mismos y con todo lo que piensan y realizan; es un procedimiento reflexivo en que el alumno se interroga sobre sus pensamientos o acciones (p. 37).

Para Barnett (1992), la competencia reflexiva vendría siendo la misma práctica reflexiva pero añadiendo un aspecto metodológico e investigativo. Es decir, la conscientización de la práctica ya no es el centro de atención sino que, además, se interrelaciona con características de la persona y su formación académica, la situación y la respuesta que va surgiendo en un proceso reflexivo sistemático e intencional.

Fourés, Pozas y López (2013), determinan que la práctica reflexiva es un proceso complejo que involucra características del docente y su quehacer pedagógico. Por lo tanto, para determinar el buen desarrollo de esta metodología es necesario una adecuada herramienta valorativa y evaluativa que permita «reconocer en los docentes un papel activo, con capacidad de pensar y formular los propósitos y finalidades de su trabajo, como así también ser generador de una actitud cuestionadora de su propia práctica» (p. 72).

\section{UNA EXPERIENCIA DE PRÁCTICA REFLEXIVA COLECTIVA EN EL CONTEXTO UNIVERSITARIO}

En educación superior, la actividad docente implica la articulación de conocimiento disciplinar y pedagógico. Sin embargo, por lo general, los profesores universitarios son solo expertos en áreas disciplinares, preparados para la producción de conocimiento en el desarrollo 
de procesos de investigación y no necesariamente tienen formación pedagógica para desarrollar el rol como docentes.

Frente a este escenario, en las instituciones de educación superior se evidencia la necesidad de apoyar a los profesores en sus procesos de formación pedagógica, mediante la oferta permanente de programas o actividades, enmarcadas en su realidad como docentes universitarios responsables de múltiples procesos.

En 2012, la Universidad del Norte crea el Centro para la Excelencia Docente (CEDU), con el propósito de apoyar a la comunidad en el desarrollo y fortalecimiento de su quehacer pedagógico, ofreciendo escenarios de formación docente en el contexto de programas, servicios y recursos destinados a fomentar la excelencia e innovación en la enseñanza, para facilitar el aprendizaje efectivo en el estudiante, de acuerdo con las tendencias que proponen investigaciones en educación superior.

Entre esta oferta de actividades para la formación docente, se presenta «Hablemos docencia», como un espacio de diálogo, que propicia la reflexión e intercambio de experiencias entre los participantes, sobre la práctica de aula en temas específicos, relacionados con la práctica pedagógica universitaria.

«Hablemos docencia» constituye un encuentro periódico, a la hora del almuerzo, donde a través de la interacción entre pares sobre un tema pedagógico, de interés común a los asistentes, los profesores reflexionan sobre su práctica docente de manera propositiva, amplían su conocimiento pedagógico o tienen un primer acercamiento sobre estrategias pedagógicas que, eventualmente, pudieran incorporar en su práctica de aula.

Actualmente, esta actividad es semanal y en horas de almuerzo; el tema es seleccionado previamente mediante votación por los docentes participantes. Cada docente inscrito recibe por correo electónico el material de lectura de máximo cuatro páginas, asociado al tema seleccionado. 
Las sesiones se enmarcan en la implementación de los incidentes críticos como estrategia para activar y movilizar las creencias y emociones de los profesores participantes, en relación con su práctica de aula e identidad docente (Monereo, 2012). Bajo este escenario se establece una dinámica de interacción; los incidentes críticos se plantean mediante el uso de fichas que expresan preguntas de reflexión, una situación o caso, una instrucción o la descripción de una estrategia pedagógica sobre el tema seleccionado que es desarrollado en la lectura. Cada docente participa de acuerdo con las indicaciones expresadas en sus fichas, impulsándose así varias rondas. El resto de docentes aporta con sus experiencias o inquietudes, y se genera una alta participación alrededor del tema tratado.

En cuanto a la participación, por período académico se mantiene un promedio de 79 docentes, distribuidos en grupos entre 12 y 15 por sesión. En total han participado, aproximadamente, 474 docentes de Uninorte en un período de seis semestres (2015-2017). Las temáticas de mayor demanda fueron: interacción social y emocional en el aula para dinamizar el aprendizaje, y estrategias para valorar el aprendizaje.

Teniendo en cuenta la metodología empleada en «Hablemos docencia» - como estrategia de práctica reflexiva colectiva-, se pretende determinar su incidencia en la percepción de los profesores participantes, en cuanto a su práctica de aula. Para ello, se realiza una caracterización de las reflexiones que expresaron algunos docentes en función a los focos técnico, contextual y de reflexividad crítica, propuestos por Tsangaridou y O'Sullivan (1994).

\section{Metodología}

El estudio se realizó bajo un enfoque de investigación cuantitativa, que permite probar hipótesis a partir de la recolección de datos, con base en la medición numérica y su análisis estadístico (Hernández, Fernández y Baptista, 2010). El tipo de la investigación es no experimental ex post facto que corresponde a un proceso investigativo, realizado sin manipular deliberadamente variables y en los que 
solo se observan los fenómenos en su ambiente natural. Después se analizan (Hernández et al., 2010) y establecen inferencias sobre las relaciones causa-efecto entre variables, sin intervención directa o influencia directa, puesto que el evento a investigar sucedió en un espacio y tiempo determinados (Bernardo y Caldero, 2000, citado por Cancela, Cea, Galindo y Valilla, 2010).

En el contexto de este estudio, las variables a analizar corresponden a la actividad «Hablemos docencia», como variable independiente, y la práctica reflexiva de los docentes, como variable dependiente. En la siguiente tabla se observa la caracterización de las variables de acuerdo con su definición conceptual y operacional.

\section{Tabla 1. Caracterización variables de la práctica reflexiva colectiva Hablemos docencia.}

\begin{tabular}{|c|c|c|}
\hline Variable & Definición conceptual & $\begin{array}{l}\text { Definición } \\
\text { operacional }\end{array}$ \\
\hline $\begin{array}{l}\text { Independiente: } \\
\text { Hablemos } \\
\text { docencia. }\end{array}$ & $\begin{array}{l}\text { Hablemos docencia: Es una } \\
\text { actividad coordinada por } \\
\text { la Unidad de Formación } \\
\text { Pedagógica Docente del CEDU, } \\
\text { que desarrolla espacios de } \\
\text { diálogo entre la comunidad } \\
\text { docente sobre temas } \\
\text { relacionados a la práctica } \\
\text { pedagógica universitaria que } \\
\text { conlleven a la reflexión e } \\
\text { intercambio de experiencias } \\
\text { sobre la labor docente. }\end{array}$ & $\begin{array}{l}\text { La asistencia es al } \\
\text { menos de cuatro } \\
\text { sesiones y máximo } \\
\text { de siete sesiones. }\end{array}$ \\
\hline
\end{tabular}




\begin{tabular}{|c|c|c|}
\hline $\begin{array}{l}\text { Dependiente: La } \\
\text { práctica reflexiva } \\
\text { de los docentes. }\end{array}$ & $\begin{array}{l}\text { Práctica reflexiva: Aprender } \\
\text { mientras se realiza una acción, } \\
\text { es decir, realizar cambios que } \\
\text { emergen como producto de } \\
\text { una concientización sobre la } \\
\text { actividad que se realiza (Dewey, } \\
\text { 1967, 1903, 1997). } \\
\text { La práctica reflexiva debe } \\
\text { contemplar los cuatro } \\
\text { elementos que son parte } \\
\text { del proceso enseñanza- } \\
\text { aprendizaje: objetivos, } \\
\text { contenidos, actividades de } \\
\text { enseñanza y evaluación } \\
\text { (Ortega, Nocetti, y Ortiz, 2015). }\end{array}$ & $\begin{array}{l}\text { Reconocimiento de } \\
\text { cambios en: } \\
\text { _ Frecuencia con } \\
\text { que el docente } \\
\text { revisa/replantea } \\
\text { su propia } \\
\text { planeación de } \\
\text { clase. } \\
\text { Frecuencia con } \\
\text { que el docente } \\
\text { revisa/replantea } \\
\text { su metodología } \\
\text { de clase. } \\
\\
\text { Grado en que } \\
\text { el docente } \\
\text { considera } \\
\text { los factores } \\
\text { individuales } \\
\text { del estudiantes } \\
\text { (edad, estilo de } \\
\text { aprendizaje, } \\
\text { contexto social) } \\
\text { para diseñar los } \\
\text { ambientes de } \\
\text { aprendizaje. }\end{array}$ \\
\hline
\end{tabular}

Fuente: Elaboración propia.

\section{Población y Muestra}

La población a investigar son los docentes que participaron en el servicio «Hablemos docencia» en los períodos académicos 2015 a 2017. Se empleó un muestreo no probabilístico, es decir, la selección de la muestra no se realiza al azar, y la selección de los participantes 
corresponde a unos criterios establecidos por el sujeto investigador, de acuerdo con los propósitos establecidos en la investigación (Hernández et al., 2010).

Se establecen como criterios de inclusión los siguientes:

1. Estar vinculados a la Universidad del Norte como docentes.

2. Haber participado en mínimo cuatro sesiones de «Hablemos docencia», durante los períodos 2015-2017.

Un total de 34 profesores participantes cumplieron con estos criterios y solo cinco de ellos dieron su consentimiento para participar en el estudio.

En la tabla 2 se caracteriza la muestra en función al género y tipo de vinculación a la institución universitaria.

Tabla 2. Caracterización muestra profesores participantes.

\begin{tabular}{|c|c|c|c|c|}
\hline \multirow{2}{*}{$\begin{array}{c}\text { Total profesores } \\
\text { participantes } \\
\text { entrevistados }\end{array}$} & \multicolumn{2}{|c|}{ Género } & \multicolumn{2}{c|}{ Vinculación } \\
\cline { 2 - 5 } & F & M & $\begin{array}{c}\text { Tiempo completo } \\
\text { (TC) }\end{array}$ & $\begin{array}{c}\text { Tiempo } \\
\text { parcial }\end{array}$ \\
\hline 5 & 2 & 3 & 2 & 3 \\
\hline
\end{tabular}

Fuente: Elaboración propia.

\section{Técnicas e Instrumentos}

Para la recolección de información, teniendo en cuenta el propósito de este estudio, se desarrollaron entrevistas (técnica cualitativa que da cuenta de la realidad de los sujetos participantes) con el apoyo de un cuestionario. Como señala Kvale (2011), «las entrevistas son adecuadas 
para estudiar la comprensión de las personas de los significados de su mundo vivido, describir sus experiencias y su modo de comprenderse a sí mismos, y aclarar y elaborar su propia perspectiva sobre su mundo vivido» (p. 73).

Para el análisis de la información correspondiente a la transcripción de las entrevistas, se empleó como técnica el análisis de contenido, con el apoyo de una rejilla de Análisis documental, que permite la clasificación y / o codificación de las las ideas expresadas en categorías, con el propósito de darle un sentido científico al propósito del proceso (Gómez Mendoza, 2000).

\section{Tabla 3. Caracterización técnica e instrumento utilizados para el estudio.}

\begin{tabular}{|l|c|c|}
\hline \multicolumn{1}{|c|}{ Variables } & Técnica & Instrumento \\
\hline $\begin{array}{l}\text { Focos y niveles de la } \\
\text { reflexión en y sobre } \\
\text { la práctica de aula. }\end{array}$ & Entrevista. & $\begin{array}{c}\text { Formato de entrevista } \\
\text { semiestructurada. }\end{array}$ \\
\cline { 2 - 3 } & Análisis de contenido. & $\begin{array}{c}\text { Ficha de análisis } \\
\text { documental. }\end{array}$ \\
\hline
\end{tabular}

Fuente: Elaboración propia.

\section{Procedimiento}

A la muestra anteriormente descrita se le aplicó un cuestionario, bajo el contexto de una entrevista semiestructurada, para indagar sobre los focos de reflexión de los docentes (técnico, contextual y reflexividad crítica) y conocer en profundidad su nivel de reflexión (descriptivo, justificativo y crítico), en torno a aspectos de su práctica pedagógica y la incidencia de la actividad de «Hablemos docencia» en su práctica reflexiva. 
El análisis de los resultados se realizó mediante la aplicación de la técnica Análisis de contenido con el apoyo de una ficha de análisis documental para la organización e interpretación de las transcripciones de los diálogos desarrollados en las entrevistas. Con esta técnica se logró la identificación de las ideas expresadas por los docentes, relacionadas con las dimensiones consideradas por Tsangaridou y O'Sullivan (1994), descritas brevemente en la siguiente tabla:

\section{Tabla 4. Descripción dimensiones de la práctica reflexiva según Tsangaridou y O'Sullivan (1994).}

\begin{tabular}{|l|l|}
\hline Dimensión técnica & $\begin{array}{l}\text { Consiste en la aplicación de conocimientos } \\
\text { (instrucciones) para la enseñanza de un material. }\end{array}$ \\
\hline Dimensión contextual & $\begin{array}{l}\text { Radica en considerar los factores situacionales } \\
\text { para el desarrollo de una clase. }\end{array}$ \\
\hline $\begin{array}{l}\text { Dimensión reflexividad } \\
\text { crítica }\end{array}$ & $\begin{array}{l}\text { Corresponde a las acciones docentes teniendo } \\
\text { en cuenta los conocimientos, los factores } \\
\text { situacionales y la propia persona. }\end{array}$ \\
\hline
\end{tabular}

Fuente: Elaboración propia.

\section{Resultados}

En la gráfica 1, puede observarse, de manera general, los resultados de la dinámica reflexiva en los profesores participantes en «Hablemos docencia», para cada uno de los focos reflexivos, propuestos por Tsangaridou y O'Sullivan (1994). De acuerdo con estos resultados, los focos reflexivos que se activan significativamente en los docentes, corresponden al contextual y técnico. 


\section{Gráfica 1. Focos de reflexión en los docentes participantes en Hablemos docencia.}

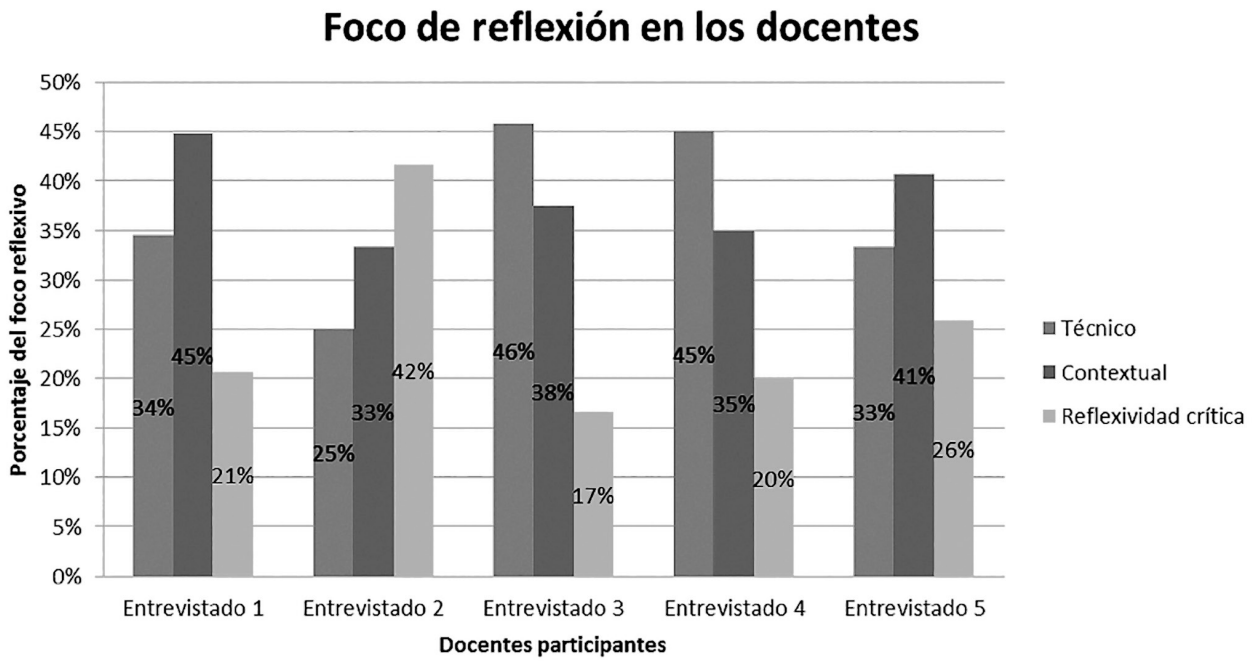

Fuente: Elaboración propia.

El foco predominante corresponde al contextual, relacionado con los factores situacionales para el desarrollo de las clases, que involucra la planificación y la selección de estrategias de aprendizaje y de evaluación que favorezcan el propósito del proceso de enseñanza-aprendizaje.

En segunda instancia, el foco técnico, corresponde a los aspectos relacionados con las instrucciones específicas que se comparten con los estudiantes para el desarrollo de alguna actividad, al diseño de materiales de estudio pertinentes de acuerdo con el desarrollo didáctico de las metodologías pedagógicas, que posibilitan el desarrollo de las clases.

Por último, el foco reflexivo crítico —con una baja exposición en comparación con los dos primeros-, comprende la aplicación de los 
conocimientos de manera justificada, teniendo en cuenta los factores situacionales y los factores individuales de los docentes (creencias, percepciones y emociones), en función a la comprensión de los principios pedagógicos que deben prevalecer para la creación de ambientes de aprendizaje.

En la gráfica 2 se muestra la frecuencia de aparición, en porcentajes, de los comentarios de los profesores participantes, teniendo en cuenta el nivel de reflexión (descriptivo, justificativo y crítico) en correspondencia al foco de reflexión (técnico, contextual y reflexividad crítica) expuesto anteriormente, con la finalidad de caracterizar la práctica reflexiva de los docentes en el contexto de la actividad de «Hablemos docencia» en función a estas dimensiones.

\section{Gráfica 2. Distribución de comentarios de los profesores participantes en Hablemos docencia.}

\section{Porcentaje de reflexión en los docentes}

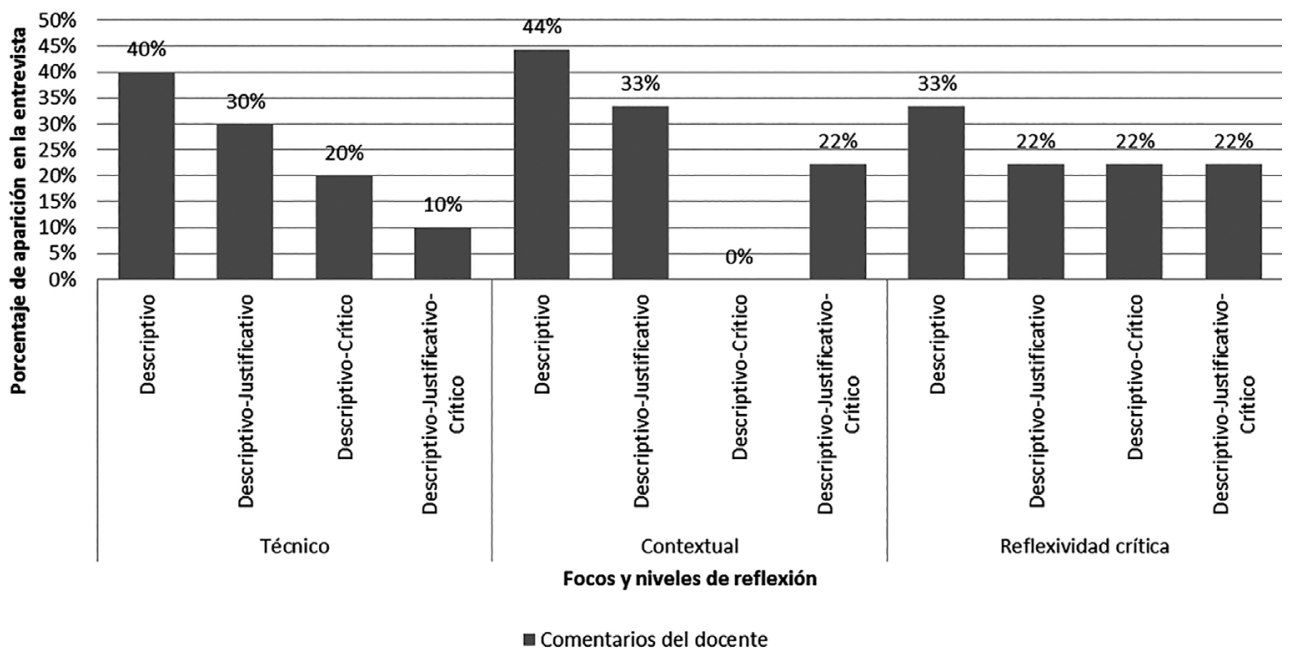

Fuente: Elaboración propia. 
De acuerto a estos resultados, en los tres focos de reflexión, el nivel descriptivo fue predominante, y corresponde a que los docentes logran desarrollar de manera significativa reflexiones relacionadas con las acciones que realizan en su práctica de aula, en relación con: planificación de su asignatura, reconocimiento de los retos que se les presentan cada semestre y cómo enseñar los contenidos de su clase.

El segundo nivel representativo corresponde al descriptivo-justificativo, relacionado con la capacidad que expresan los docentes participantes en la actividad de «Hablemos docencia» en identificar lo que proponen en sus clases, basados en un ensayo-error y en precisar los factores que pueden influir en el desarrollo del proceso de enseñanza-aprendizaje.

El nivel descriptivo-justificativo-crítico, como tercer aspecto sobresaliente, hace referencia a que algunos docentes emplean la reflexión de manera constante y la enriquecen no solo con la retroalimentación de sus estudiantes a las clases y con los procesos de investigación de aula que han realizado, sino que reconocen los aspectos positivos de su docencia y lo que se requiere mejorar con el propósito de fortalecer su práctica de aula.

Por último, el nivel descriptivo-crítico, señala la valoración que los docentes realizan sobre su quehacer pedagógico, considerando la posibilidad de evaluar o auto evaluar su práctica para caracterizarla en sí misma. Este nivel, si bien es bajo en relación con los otros, se entiende como una característica que establece la base del nivel descriptivo-justificativo-crítico porque se relaciona con la toma de decisiones para establecer cambios que promuevan y fundamentan la innovación pedagógica.

El análisis propuesto en este apartado, confirma el propósito y las características de «Hablemos docencia» en cuanto al foco y nivel del ejercicio reflexivo en los participantes, teniendo en cuenta que la dinámica de esta estrategia propone un acercamiento inicial, a nivel descriptivo y circunstancial, a temas o aspectos pedagógicos no necesariamente familiares para los profesores que participan en esta actividad. 


\section{CONCLUSIONES}

La práctica reflexiva requiere de un propósito y una estructura que posibilite su desarrollo en el contexto de la realidad que vive el profesor. Es en la reflexión de los saberes que configuran la experiencia docente donde encontrarán los fundamentos profesionales para transformar su práctica de aula.

Es por ello que el Centro para la Excelencia Docente de la Universidad del Norte, permite el desarrollo de estas prácticas en diferentes escenarios; entre ellos se destaca la actividad de «Hablemos docencia».

Este espacio, por sus características, se ha consolidado como una valiosa estrategia de práctica reflexiva en una dinámica colectiva que involucra la participación con otros docentes, quienes se constituyen a sí mismos, en sujetos de referencia para propiciar una revisión de la experiencia docente con interlocutores válidos.

Los resultados obtenidos en este estudio evidencian el efecto de la actividad «Hablemos docencia» en cuanto a la activación y desarrollo del pensamiento reflexivo, mediante la propuesta de una metodología que permite focalizar el sentido y propósito de la reflexión. En este caso, el empleo de fichas - como artefactos mediadores-y el diálogo en comunidad - que considera los saberes, vivencias y sentir docente-, permiten la revisión in situ de la experiencia docente que puede plantear una posible ruta de trabajo para considerar, a mediano plazo, cambios en la práctica de aula.

Por el alcance de esta actividad, las características del pensamiento reflexivo que se caracterizan bajo la propuesta Tsangaridou y O'Sullivan (1994), en «Hablemos docencia», predominan el foco contextual y técnico. El primero de ellos enfatiza la reflexión, en cuanto a comprender la importancia de una identificación del alcance de las estrategias de aprendizaje y de evaluación que permiten el logro de los objetivos de aprendizaje. Este resultado corresponde al propósito de la dinámica planteada durante las sesiones de «Hablemos docencia»; porque esta actividad se enfoca en la revisión de algunos temas que proponen la revisión sobre estrategias pedagógicas que la investigación respalda para promover el aprendizaje en educación superior. 
El foco técnico, inmerso en el contextual, resalta la capacidad reflexiva de los profesores participantes en identificar los aspectos relacionados con la planeación de sus actividades y con describir lo que sucede en su práctica de aula. Algunos de ellos mostraron matices propios del foco de reflexividad crítica, al justificar y evaluar las decisiones que toman durante y sobre la acción docente.

Por último, es necesario destacar que «Hablemos docencia» se ha establecido como uno de los espacios de práctica reflexiva colectiva, con una importante participación por parte de los profesores en la Universidad del Norte. Esta actividad permite sensibilizar a la comunidad docente frente a la importancia de la práctica reflexiva como un medio para pensar y sentir la docencia de una manera significativa, con el respaldo de experiencias de y con otros.

Como centro de apoyo a la docencia, el reto se encuentra en fortalecer estos espacios con metodologías que propicien un pensamiento reflexivo estructurado y con sentido, permitiendo a los profesores encontrar el significado de su práctica de aula.

\section{REFERENCIAS}

Cancela, R., Cea, N., Galindo, G., y Valilla, S. (2010). Metodología de la investigación educativa: Investigacion Ex Post Facto. Universidad Autónoma de Madrid, 1-19.

Catalán, J. (2011). Del pensamiento al conocimiento profesional del profesor. Psicología Educacional. Rumbos, problemáticas y aportaciones, 187-215.

Catalán, J., y Castro, P. (2016). Reflexión colectiva sistemática: un estudio orientado al desarrollo profesional docente. Psicología Escolar e Educacional, 157-167.

Domingo, A. (2009). «Desarrollar la competencia reflexiva en la educación superior. Diez propuestas para el aula universitaria». Revista Panamericana de Pedagogía, 33-57. 
Domingo, A., y Gómez, M. (2014). La práctica reflexiva. Bases, Modelos e Instrumentos . España: Narcea, S.A.Ediciones.

Elías, M., Barcia, M., y Hernando, G. (2009). «Influencias en la génesis del concepto de enseñanza reflexiva». Cuadernos de Educación, 103-113.

Fourés, C., Pozas, D., y López, N. (2013). «La escritura de auto-registros como proceso de recuperación y reflexión sobre la práctica docente». Educación, Lenguaje y Sociedad, 67-78.

Gómez Mendoza, M. (2000.) «Análisis de contenido cualitativo y cuantitativo: Definición, clasificación y metodología». Revista de Ciencias Humanas, 20.

Hernández, R., Fernández, C., y Baptista, P. (2010). Metodología de la Investigación. Mexico: McGraw Hill.

Kvale, S. (2011). Las entrevistas en investigación cualitativa. Madrid: Ediciones Morata.

Monereo, C.; Panadero E. y Scartezini, R. (2013). «SharEVents. La utilización de informes compartidos sobre incidentes críticos como medio para la formación docente». Cuadernos de Educación, 45-67

Ortega, J., Nocetti, A., y Ortiz, L. (2015). «Prácticas reflexivas del proceso de enseñanza en docentes universitarios de las ciencias de la salud». Educación Médica Superior, 576-590.

Schön, D. (1987). La formación de profesionales reflexivos. Hacia un nuevo diseño de la enseñanza y el aprendizaje en las profesiones. Paidos.

Serrano, J. (2005). Reseña de «Cómo pensamos. Nueva exposición de la relación entre pensamiento reflexivo y proceso educativo de John Dewey». Intercontinental de Psicología y Educación, 154-162.

Tsangaridou, N., \& O'Sullivan, M. (1994). «Using Pedagogical Reflective Strategies to Enhance Reflection Among Preservice Physical Education Teachers». Journal of Teaching in Physical Education, 13-33. 